PROCEEDINGS OF THE

AMERICAN MATHEMATICAL SOCIETY

Volume 131, Number 10, Pages 3267-3274

S 0002-9939(03)06926-0

Article electronically published on February 12, 2003

\title{
MEAN VALUE EXTENSION THEOREMS AND MICROLOCAL ANALYSIS
}

\author{
ERIC TODD QUINTO
}

(Communicated by Andreas Seeger)

\begin{abstract}
We use microlocal analysis to prove new mean value theorems for harmonic functions on harmonic manifolds and for solutions to more general differential equations. The equations we consider all satisfy spherical mean value equalities, at least locally. Microlocal analysis and the mean value property in a small set allows us to show that the solution to the differential equation in a small set is also a solution in a much larger set.
\end{abstract}

\section{INTRODUCTION}

The fundamental mean value theorem in $\mathbb{R}^{n}$ characterizes harmonic functions by their averages over spheres. Many generalizations have been proven, including extensions to more differential equations, other manifolds, and weakening of hypotheses. In this article, we will use microlocal analysis and classical results, including Pizzetti's formula and generalizations, to prove mean value extension theorems on manifolds.

The classical mean value theorem states that $f \in C\left(\mathbb{R}^{n}\right)$ is harmonic if and only if for all $x \in \mathbb{R}^{n}$ and all $r>0$,

$$
S M f(x, r):=\frac{1}{\operatorname{Vol}(S(x, r))} \int_{y \in S(x, r)} f(y) d y=f(x) .
$$

where $d(x, y)=\|x-y\|$ is the standard distance, $S(x, r)=\left\{y \in \mathbb{R}^{n} \mid d(x, y)=r\right\}$ is the sphere centered at $x$ and of radius $r$, and $d y$ is the geodesic measure on $S(x, r) ; S M$ is just the spherical mean of $f$ over $S(x, r)$. This equivalence between (1.1) and harmonicity is valid if (1.1) is true for two well chosen radii, [12, 27, and this two-radius theorem has been generalized to rank one symmetric spaces 6, 8, 22, and to other differential equations [10, 21]. One can also prove mean value characterizations of harmonic functions with restricted sets of centers and arbitrary radius [1, 2].

Generalizations have been made to different partial differential equations (PDE) in $\mathbb{R}^{n}$ by Poritsky [19], Volchkov [21] and others. Zalcman proved a precise correspondence between solutions to differential equations and generalized mean value theorems [26]: a continuous function in $\mathbb{R}^{n}$ satisfies a generalized mean value theorem if and only if it is a solution to associated PDEs.

Received by the editors May 1, 2002.

2000 Mathematics Subject Classification. Primary 58J05, 44A12; Secondary 35J05, 58J40.

The author was partially supported by NSF grant 9877155 and later by grant 0200788 . 
Generalized mean value theorems can be proven using Pizzetti's formula [17, 18, and generalizations [26, 7] 14, as we will do in 4.1, Pizzetti's formula can be put into a very general context using spectral theory (see the proofs of [8, (7.5)] and [14, Theorem 2.7, p. 95], and the article [20]). Characterizations of pluriharmonic and separately harmonic function by mean values over ellipsoids and distinguished boundaries of polydisks have been proven 3 . Numerous results and references to related problems are given in the well written overview articles [5, 27, 28, 29].

In the next section we will prove mean value extension theorems for harmonic functions on manifolds. Extension theorems are proven for solutions to other PDEs on $\mathbb{R}^{n}$ in $\$ 3$, and on symmetric spaces in $\$ 4$

\section{HARMONIC FUNCTIONS}

The basic mean value theorem is the equivalence of (1.1) and harmonicity, and the most general setting in which this is valid is that of harmonic manifolds [25]. pp. $223 \mathrm{ff}$.$] .$

Definition 2.1. A smooth Riemannian manifold without boundary, $M$, is locally harmonic (or a harmonic space) if and only if for each $x \in M$ there is a positive real number $\epsilon$ and a function $G:[0, \epsilon) \rightarrow \mathbb{R}$ such that the function $g: D(x, \epsilon) \backslash\{x\} \rightarrow \mathbb{R}$ defined by $g(y)=G(d(x, y))$ satisfies $\Delta g=0$.

The harmonic manifolds include all two-point homogeneous spaces (spaces for which there is an isometry taking any two points to any other two points the same distance apart). These are the rank-one symmetric spaces. However, contrary to the classical conjecture of Lichnerowicz, there is a class of harmonic spaces, the Damek-Ricci spaces, that are not two-point homogeneous spaces. See [9] for details.

Let $M$ be a Riemannian manifold. Then, the left-hand side of (1.1) makes sense where $S(x, r)$ is the geodesic sphere centered at $x \in M$ and of sufficiently small radius $r>0$. Let $D(x, r)=\{y \in M \mid d(x, y) \leq r\}$ be the closed disk of radius $r$ centered at $x$ and for $U \subset M$, let $D(U, r)=\bigcup_{x \in U} D(x, r)$.

A fundamental result of Willmore [23] states that every harmonic function in a harmonic space satisfies (1.1), at least for spheres of small radius. Furthermore, if a $C^{2}$ function satisfies (1.1) locally about each point in a harmonic manifold, then it is harmonic [25. Theorem 6.7.6]. Finally, it is useful to note that every locally harmonic manifold is real-analytic. This is true because harmonic manifolds are Einstein and Einstein manifolds are real-analytic [25, pp. 229-230].

These basic theorems and microlocal analysis allow us to prove the following mean value extension theorem.

Theorem 2.2. Let $M$ be a locally harmonic manifold and let $U$ be a nonempty connected open set in $M$. Let $R>0$ and assume for each $y \in D(U, 2 R)$ the injectivity radius of $M$ at $y$ is greater than $R$. Assume $f \in C^{2}(D(U, R))$ and for each $x \in U$ and each $r \in(0, R), f$ satisfies the mean value equality (1.1). Then $f$ is harmonic in $D(U, R)$.

On the other hand, if $f$ is harmonic in $D(U, R)$, then $f$ satisfies the mean value equality (1.1) for all $x \in U$ and $r \in(0, R)$.

In the first part of this theorem, we assume the mean value property only for spheres with center in the small set $U$, and our theorem allows us to extend harmonicity out of $U$ into all of $D(U, R)$. Even on $\mathbb{R}^{n}$, classical counterexamples 
show the hypothesis $f \in C^{2}$ is necessary for the first part of the theorem. The assumptions about injectivity radius ensure that spheres $S(y, r)$ will be diffeomorphic to Euclidean spheres and that $S M$ and its dual will be well defined real-analytic Fourier integral operators. The second part of the theorem is classical.

Proof of Theorem 2.2. We need two theorems from real-analytic microlocal analysis. For a distribution, $f$ on $M$, let $\mathrm{WF}_{\mathrm{A}}(f)$ be the real-analytic wavefront set of $f$ [15. Definition 8.4.3]; intuitively, $(x, \xi) \in \mathrm{WF}_{\mathrm{A}}(f)$ means that $f$ is not real-analytic at $x$ in direction $\xi \in T_{x}^{*}(M)$.

Let $W$ be a submanifold of $M$, then the conormal bundle $N^{*}(W)$ is defined as the set of all covectors above $W$ that are conormal to $T(W): N^{*}(W)=\{(x, \xi) \in$ $\left.T^{*}(M) \mid x \in W, T_{x}(W) \subset \operatorname{ker} \xi\right\}$. The first theorem we need is a result of Kawai, Kashiwara, and Hörmander.

Lemma 2.3 (Microlocal Holmgren Lemma [15. Theorem 8.5.6]). Let $M$ be a realanalytic manifold and let $W$ be a $C^{2}$ hypersurface in $M$. Let $f$ be a distribution in M. Let $\left(y_{0}, \xi_{0}\right) \in N^{*}(W) \backslash\{0\}$ and assume $y_{0} \in \operatorname{supp} f$ and $f$ is zero locally near $y_{0}$ on one side of $W$ Then $\left(y_{0}, \xi_{0}\right) \in \mathrm{WF}_{\mathrm{A}}(f)$.

If $f$ is zero on one side of $W$ near $y_{0}$ and $y_{0} \in \operatorname{supp} f$, then $f$ cannot be realanalytic near $y_{0}$. Lemma 2.3 is a strengthening of this idea because it asserts not only that $f$ is not real-analytic at $y_{0}$, but that the conormal directions to $W$ must be in $\mathrm{WF}_{\mathrm{A}}(f)$ above $y_{0}$.

The other fundamental theorem is a microlocal regularity theorem for $S M$. Under the assumptions of Theorem 2.2, $S M$ is a real-analytic elliptic Fourier integral operator associated to a specific Lagrangian manifold, and the microlocal analysis of this operator was worked out in [13, §3.2]. The microlocal regularity lemma is as follows.

Lemma 2.4 (Microlocal Regularity Lemma [13, Proposition 3.2.1]). Let $M$ be a real-analytic manifold. Let $R>0$ and assume for each $y \in D(U, 2 R)$ the injectivity radius of $M$ at $y$ is greater than $R$. Let $f$ be a distribution in $M$ and assume $\operatorname{SMf}(x, r)$ is real-analytic for $(x, r) \in U \times(0, R)$. Then

$$
\mathrm{WF}_{\mathrm{A}}(f) \cap N^{*}(S(x, r))=\emptyset \quad \forall x \in U, r \in(0, R) .
$$

What (2.1) means is that if $S M f$ is real analytic, then $f$ must be real analytic in directions conormal to the spheres, $S(x, r)$, over which spherical mean integrates. The proof of Lemma 2.4 is given in [13], and it includes a calculation of the Lagrangian manifold associated to the Fourier integral operator $S M$.

Our last tool shows that a zero function extends if the analyticity condition (2.1) holds.

Lemma 2.5 (Extension Lemma). Let $M$ be a real-analytic manifold and let $U$ be a nonempty connected open set in $M$. Let $R>0$ and assume for each $y \in D(U, 2 R)$ that the injectivity radius of $M$ at $y$ is greater than $R$. Assume $g$ is a distribution in $D(U, R)$ and for each $x \in U$ and each $r \in(0, R), g$ satisfies the regularity condition (2.1). Assume also that $g$ is zero in $U$. Then, $g$ is zero in the larger set $D(U, R)$.

\footnotetext{
${ }^{1}$ There is an open neighborhood, $V$, of $y_{0}$ such that $W \cap V$ divides $V$ into two disjoint open sets, and $f$ is zero on one of these sets.
} 
Proof. Let $x_{0} \in U$ and let $r_{0} \in[0, R]$ be the smallest radius such that $S\left(x_{0}, r_{0}\right)$ meets $\operatorname{supp} g ; r_{0}>0$ because $g$ is zero in $U$. We will show $r_{0}=R$. Assume $r_{0} \in(0, R)$. Let $y_{0}$ be one of the points of intersection of $\operatorname{supp} g$ and $S\left(x_{0}, r_{0}\right)$, and let $\xi_{0} \in N_{y_{0}}^{*}\left(S\left(x_{0}, r_{0}\right)\right) \backslash\{0\}$.

Because $g$ is zero inside $S\left(x_{0}, r_{0}\right)$ and $y_{0} \in(\operatorname{supp} g) \cap S\left(x_{0}, r_{0}\right),\left(y_{0}, \xi_{0}\right) \in \mathrm{WF}_{\mathrm{A}}(g)$ by the Microlocal Holmgren Lemma 2.3. However, by assumption (2.1),$\left(y_{0}, \xi_{0}\right) \notin$ $\mathrm{WF}_{\mathrm{A}}(g)$. This proves by contradiction that $r \geq R$, and the lemma is proven.

Now we have the tools to prove Theorem 2.2 Assume $f$ satisfies (1.1) for all $x \in U$ and $r \in(0, R)$. Then, by [25] Theorem 6.7.6], $f$ is harmonic in $U$ since the mean value property holds for all spheres in $U$. Hence $f$ is real-analytic in $U$. Then, by the mean value equality (1.1), which is valid for $x \in U$ and $r \in(0, R)$, $S M f(x, r)=f(x)$ is real-analytic for $(x, r) \in U \times(0, R)$. The Microlocal Regularity Lemma 2.4 can be used to conclude that (2.1) holds for $f$ and therefore for $\Delta f$ since $\Delta$ is a real-analytic differential operator. Finally, the Extension Lemma 2.5 allows us to show that $\Delta f=0$ in $D(U, R)$ since $\Delta f=0$ in $U$.

The second implication of Theorem 2.2 follows from Willmore's fundamental result 23 which states that a harmonic function in an open disk in a harmonic manifold satisfies the mean value theorem for any sphere in the set.

One can use the Microlocal Regularity Lemma 2.4 and the Extension Lemma 2.5. as above, to provide a new support theorem for the sphere transform. The theorem is new even in $\mathbb{R}^{n}$, but a classical proof can be given for $\mathbb{R}^{n}$.

Theorem 2.6. Let $M$ be a real-analytic manifold and let $U$ be a nonempty connected, open set in $M$. Let $R>0$ and assume for each $y \in D(U, 2 R)$ the injectivity radius of $M$ at $y$ is greater than $R$. Assume $f$ is a distribution in $D(U, R)$ and for each $x \in U$ and each $r \in(0, R), S M f(x, r)=0$. If $f$ is zero in $U$, then $f$ is zero in $D(U, R)$.

\section{OTHER DIFFERENTIAL EQUATIONS}

In this short section, we apply the proof methods of $\$ 2$ to a mean value theorem for the Helmholtz equation and for polyharmonic functions in $\mathbb{R}^{n}$. One can prove these theorems by first proving Theorem 2.6 in $\mathbb{R}^{n}$ using classical techniques, but our ideas apply immediately to these equations, and they suggest theorems for other PDEs

It is well known [11 p. 289] that if $U \subset \mathbb{R}^{n}$ is an open disk and $f \in C^{2}(U)$, then $f$ satisfies the Helmholtz equation

$$
\Delta f+\lambda f=0
$$

if and only if

$$
S M f(x, r)=\Gamma(n / 2) \frac{J_{(n-2) / 2}(r \sqrt{\lambda})}{(r \sqrt{\lambda} / 2)^{(n-2) / 2}} f(x)
$$

for all spheres contained in $U$ [11]. Here $J_{(n-2) / 2}$ is a Bessel function of the first kind. Two radius mean value theorems have been proven for (3.1) by Volchkov [21], and Chamberland [10], and related theorems were proven in [19, 26, 27]. 
Our mean value extension theorem for the Helmholtz equation is the following.

Theorem 3.1. Let $U$ be a nonempty connected, open set in $\mathbb{R}^{n}$. Let $R>0$ and assume $f \in C^{2}(D(U, R))$ satisfies the mean value property (3.2) for each $x \in U$ and each $r \in(0, R)$. Then $f$ satisfies the Helmholtz Equation (3.1) in $D(U, R)$.

On the other hand, if $f$ satisfies the Helmholtz Equation in $D(U, R)$, then $f$ satisfies the mean value equality (3.2) for all $x \in U$ and $r \in(0, R)$.

Proof. The proof is similar to the proof in $\$ 2$ and it will be outlined. Assume that $f \in C^{2}(D(U, R))$ satisfies the mean value property (3.2) for every $x \in U$ and $r \in(0, R)$. By [19], $f$ satisfies the Helmholtz equation in $U$. Therefore, $f$ is real-analytic in $U$. By 3.2 and because $J_{(n-2) / 2}$ is real-analytic, this shows $S M f(x, r)$ is real analytic for each $x \in U$ and each $r \in(0, R)$. By the Microlocal Regularity Lemma 2.4 and since $(\Delta+\lambda)$ is an analytic partial differential operator, $\mathrm{WF}_{\mathrm{A}}((\Delta+\lambda) f) \cap N^{*}(S(x, r))=\emptyset$ for all $x \in U, r \in(0, R)$. The proof is finished using the Extension Lemma 2.5 on $(\Delta+\lambda) f$. The second implication is classical [11, p. 289].

This theorem is really about eigenfunctions of the Laplacian, and Fulton Gonzalez and the author are developing an appropriate generalization to symmetric spaces.

The key to the proof of Theorem [3.1 can be applied whenever there is a generalized mean value theorem. Using results of Poritsky [19] (see also [11]), we prove theorems for polyharmonic functions. We use the following lemma.

Lemma $3.2(19,11)$. Let $N \in \mathbb{N}$, and let $D$ be an open disk in $\mathbb{R}^{n}$. Let $f \in$ $C^{2 N}(D)$. Then, $\Delta^{N} f=0$ on $V$ if and only if the mean value theorem

$$
S M f(x, r)=\Gamma(n / 2) \sum_{m=0}^{N-1} \frac{r^{2 m} \Delta^{m} f(x)}{2^{m} m ! \Gamma\left(\frac{n}{2}+m\right)}
$$

holds for all spheres $S(x, r) \in D$.

We use this lemma to prove the following mean value extension theorem.

Theorem 3.3. Let $U$ be a connected open set in $\mathbb{R}^{n}$ and let $N \in \mathbb{N}$. Let $R>0$ and let $f$ be a $C^{2 N}$ function in $D(U, R)$. Assume that $f$ satisfies the mean value equality (3.3) for all $x \in U$ and $r \in(0, R)$. Then, $f$ satisfies $\Delta^{N} f=0$ in all of $D(U, R)$.

On the other hand, if $f$ satisfies $\Delta^{N} f=0$ in $D(U, R)$, then $f$ satisfies (4.2) for $x \in U$ and $r \in(0, R)$.

Proof. The proof of the first implication is just as the proofs above, using the fact that Lemma 3.2 implies that $\Delta^{N} f=0$ in $U$, so $f$ is real-analytic in $U$. Then (3.3) shows $S M f$ is real-analytic in $U \times(0, R)$, so the Microlocal Regularity Lemma 2.4 can be used. Finally, we use the Extension Lemma 2.5. The other implication follows from Lemma 3.2 .

\section{Pizzetti's Formula AND Generalized MeAn VAlue theOrEms}

Pizzetti's formula [17, 18] relates the circular mean value of a real-analytic function in the plane to an infinite sum of derivatives of the function at the center. It has been generalized to spherical mean values in $\mathbb{R}^{n}$ [10, p. 287], [16, 19, 26] and 
symmetric spaces ([8] for rank one, [14, Theorem 2.7, p. 95] in general) and a generalization to manifolds was stated in [24. Morally, Pizzetti's formula is true because the mean value operator is a function of the Laplacian (or bi-invariant differential operators) (see [14] and [20]).

We will prove a generalized mean value theorem for non-compact two-point homogeneous spaces (rank-one symmetric spaces). Let $M$ be a non-compact rank-one symmetric space and let $U \subset M$ be open and connected. Let $f$ be a real-analytic function on $U$. Then, Pizzetti's formula [8, (7.5)] states that for $r$ sufficiently close to zero,

$$
\begin{gathered}
S M f(x, r)=\Gamma(n / 2) \sum_{m=0}^{\infty}\left(\frac{\sinh \kappa r}{2 \kappa}\right)^{2 m} L_{m} f(x) \text { where } \\
L_{m}=\frac{\Delta\left(\Delta-(2 n+4 \beta+4) \kappa^{2}\right) \cdots\left(\Delta-(m-1)(2 n+4 \beta+4 m-4) \kappa^{2}\right)}{m ! \Gamma(m+(n / 2))}
\end{gathered}
$$

where $n$ is the dimension of $M$ and where $\beta$ and $\kappa$ are constants related to the structure and curvature of $M$ [8]. It should be pointed out that the radius of convergence in $r$ of (4.1) can be arbitrarily small [4.

The following lemma follows immediately from (4.1).

Lemma 4.1. Let $M$ be a non-compact rank one symmetric space and let $U \subset M$ be open and connected. Assume that $f \in C^{2 N}(U)$ and $L_{N} f=0$ in $U$. Then, $f$ is real-analytic in $U$ and $f$ satisfies the mean value equality

$$
S M f(x, r)=\Gamma(n / 2) \sum_{m=0}^{N-1}\left(\frac{\sinh \kappa r}{2 \kappa}\right)^{2 m} L_{m} f(x)
$$

for $x \in U$ and for sufficiently small $r$.

On the other hand, if $f$ is real-analytic and satisfies (4.2) for $x \in U$ and for sufficiently small $r$, then $L_{N} f=0$ in $U$.

Proof. For the first implication, we note that since $L_{N} f=0$ in $U$ and since $L_{N}$ is analytic elliptic, then $f$ is real-analytic in $U$. Furthermore, since $L_{N}$ is a factor of $L_{k}$ for $k \geq N, L_{k} f=0$ for $k \geq N$. Now, equation (4.2) follows immediately from Pizzetti's formula (4.1). On the other hand, assume $f$ satisfies (4.2) for sufficiently small $r$. Since $f$ is real-analytic, $f$ also satisfies the infinite sum (4.1) for small $r$. Therefore, for $k \geq N, L_{k} f=0$.

Theorem 4.2. Let $M$ be a non-compact rank-one symmetric space and let $U$ be a connected open set in $M$. Let $R>0$ and let $f$ be a real-analytic function in $D(U, R)$. Assume that $f$ satisfies the mean value equality (4.2) for all $x \in U$ and $r \in(0, R)$. Then, $f$ satisfies $L_{N} f=0$ in all of $D(U, R)$.

On the other hand, if $f$ satisfies $L_{N} f=0$ in $D(U, R)$, then $f$ satisfies (4.2) for $x \in U$ and $r \in(0, R)$.

Since $L_{1}=\Delta$, this gives Theorem 2.2 for non-compact rank-one symmetric spaces. Theorem 3.3 is the analogous theorem for $\mathbb{R}^{n}$. A form of Pizzetti's formula with a finite sum plus a remainder term is valid for $C^{2 N}$ functions on $\mathbb{R}^{n}[11]$ (32), p. 287]. Such a theorem should be true for symmetric space for sufficiently smooth functions, and it would allow one to relax the real-analyticity assumption in Theorem 4.2 to $f \in C^{2 N}$. 
Proof. First, assume $f$ satisfies (4.2) for $x \in U$ and $r \in(0, R)$. By Lemma 4.1 $L_{N} f=0$ for $x \in U$. Furthermore, since (4.2) holds for $r \in(0, R)$, and the righthand side of the equation (4.2) is analytic for $(x, r) \in U \times(0, R), f$ satisfies the Microlocal Regularity Lemma 2.4 as does $L_{N} f$. Now, by the Extension Lemma 2.5, we see $L_{N} f=0$ in $D(U, R)$.

The other implication is more elementary. Assume $L_{N} f=0$ in $D(U, R)$. Then, $f$ is real-analytic in $D(U, R)$. Since $S M$ is a real analytic Fourier integral operator and $\mathrm{WF}_{\mathrm{A}}(f)$ is empty above $D(U, R)$, then $\mathrm{WF}_{\mathrm{A}}(S M f)$ is empty above $U \times(0, R)$. That is, $S M f(x, r)$ is real-analytic for all $x \in U, r \in(0, R)$. Fix $x_{0} \in U$, then $S M f\left(x_{0}, r\right)$ is real-analytic as a function of $r \in(0, R)$, and it satisfies the realanalytic equation (4.2) for small $r$ by Lemma 4.1. Therefore, (4.2) must hold for $r \in(0, R)$.

\section{ACKNOWLEDGMENTS}

The author thanks Mark Agranovsky, Fulton Gonzalez, and Eric Grinberg for astute observations about this research and for many lively discussions over the years.

\section{REFERENCES}

[1] M. Agranovsky and E.T. Quinto, Injectivity sets for the Radon transform over circles and complete systems of radial functions, J. Funct. Anal. 139 (2), (1996), 383-414. MR 98g:58171

[2] , Injectivity of the spherical mean operator and related problems, in: Complex Analysis, Harmonic Analysis and Applications (R. Deville et al, eds.), Addison Wesley, London, 1996. MR 97m:44004

[3] L.A. Aizenberg, C.A. Berenstein, and L. Wertheim, Mean-value characterization of pluriharmonic and separately harmonic functions, Pacific J. Math., 175(1996), 295-306. MR 98i:32016

[4] D.H. Armitage and Ü. Kuran, The convergence of the Pizzetti Series in Potential Theory, J. Math. Anal. Appl., 171(1992), 516-531. MR 93k:31002

[5] C.A. Berenstein, D-C. Chang, D. Pasucas, and L. Zalcman, Variations on the theorem of Morera, Contemporary Math., 137(1992),63-78. MR 93j:32007

[6] C.A. Berenstein and D. Pascuas, Morera and Mean-value type theorems in the hyperbolic disk, Israel J. Math., 86(1994), 61-106. MR 95j:30036

[7] C.A. Berenstein and L. Zalcman, Pompeiu's problem on spaces of constant curvature, $J$. Anal. Math., 30(1976), 113-130. MR 55:11172

[8] _ Pompeiu's problem on symmetric spaces, Comment. Math. Helv., 55(1980), 593621. MR 83d:43012

[9] J. Berndt, F. Tricerri, and L. Vanhecke, Generalized Heisenberg Groups and Damek-Ricci Harmonic Spaces, Lecture Notes in Mathematics, Vol. 1598, Springer, Heidelberg, 1995. MR 97a:53068

[10] M. Chamberland, Mean value integral equations and the Helmholtz equation, Results Math. 30(1996), 39-44. MR 97f:39031

[11] R. Courant and D. Hilbert, Methods of Mathematical Physics, vol. 2, Interscience, New York, 1962. MR 25:4216

[12] J. Delsarte and J.L. Lyons, Moyennes généralisées, Comment. Math. Helv., 33(1959), 5969. MR 21:1461

[13] E.L. Grinberg and E.T. Quinto, Morera theorems for complex manifolds, J. Funct. Anal. 178(2000), 1-22. MR 2001k:53143

[14] S. Helgason, Geometric Analysis on Symmetric Spaces, Amer. Math. Soc, Providence, RI, USA, 1994. MR 96h:43009

[15] L. Hörmander, The Analysis of Linear Partial Differential Operators I, Springer Verlag, New York, 1983. MR 85g:35002a

[16] F. John, Plane Waves and Spherical Means, Interscience, New York, 1955. MR 17:746d 
[17] P. Pizzetti, Sulla media dei valori che una funzione del punti dello spazio assume alla superficie di una sfera, Rend. Lincei ser. 5, 18(1909), 182-185.

[18] Sul significato geometrico del secundo parametra differenziale di una funcione sopra una superficie qualunque, Rend. Lincei ser. 5, 18(1909), 309-316.

[19] H. Poritsky, Generalizations of the Gauss law of the spherical mean, Trans. Amer. Math. Soc., 43(1938), 199-225.

[20] R. Strichartz, Mean value properties of the Laplacian via spectral theory, Trans. Amer. Math. Soc. 284(1984), 219-228. MR 85h:31008

[21] V.V. Volchkov, New mean-value theorems for solutions of the Helmholtz Equation, translation in Russian Acad. Sci. Sb. Math. 79(1994), 281-286. MR 94h:35043

[22] V.V. Volchkov, Spherical means on symmetric spaces Dokl. NAN Ukraine (2002), 15-19.

[23] T.J. Willmore, Mean value theorems in harmonic Riemannian spaces, J. London Math. Soc., 25(1950), 54-57. MR 11:436f

[24] - An extension of Pizzetti's formula to Riemannian manifolds, Astérisque, 80(1980), 53-56. MR 82h:53060

[25] — Riemannian Geometry, Oxford University Press., 1993. MR 95e:53002

[26] L. Zalcman, Mean Values and Differential Equations, Israel J. Math., 16(1973), 339-352. MR 49:613

[27] — Offbeat Integral Geometry, Amer. Math. Monthly, 87(1980), 161-175. MR 81b:53046

[28] - A bibliographic survey of the Pompeiu problem, in Approximation of Solutions of Partial Differential Equations, eds. B. Fuglede, M. Goldstein, W. Haussmann W.K. Hayman, and L. Rogge, C: Mathematics and Physical Sciences, NATO ASI Series, vol. 365, Kluwer Academic, Boston, 1992, pp. 185-194. MR 93e:26001

[29] - Supplementary bibliography to "A bibliographic survey of the Pompeiu problem", Contemporary Math., 278(2001), 69-76.

Department of Mathematics, Tufts University, Medford, Massachusetts 02155

E-mail address: Todd.Quinto@tufts.edu 